Check for updates

Cite this: RSC Adv., 2017, 7, 54829

\title{
Highly stretchable fiber-based single-electrode triboelectric nanogenerator for wearable devices $\uparrow$
}

\author{
Jiwon Park, (D) A Young Choi, Chang Jun Lee, Dogyun Kim and Youn Tae Kim*
}

Fiber- or thread-based triboelectric nanogenerators are suitable for wearable applications such as clothes embedded with communication devices or other electronic textiles. Unfortunately, previously reported fiber-based triboelectric nanogenerators had poor stretchability, because of which they were not suitable for weaving applications. In this paper, we propose a new structure of a fiber-based singleelectrode triboelectric nanogenerator (FSTENG). The proposed FSTENG uses silicone rubber as the negative part and a conductive thread as the electrode of the TENG. The electrical output of the FSTENG is generated by the continuous contact and separation between human skin and silicone rubber. A prototype of the proposed FSTENG showed an electrical output of $28 \mathrm{~V}$ and $0.56 \mu \mathrm{A}$, when in contact with human skin, and exhibited a high strain of up to $100 \%$. In addition, we fabricated a woven structure with dimensions of $45 \mathrm{~mm} \times 45 \mathrm{~mm}$, incorporating the FSTENG, and confirmed its power generation capabilities using LEDs and an electronic watch. The proposed FSTENG can be applied to various products ranging from wearable and stretchable energy harvesters to smart clothing, by facilitating the manufacture of large textiles.

Received 16th September 2017 Accepted 26th November 2017

DOI: 10.1039/c7ra10285b

rsc.li/rsc-advances
However, triboelectric energy harvesting is a phenomenon in which energy is generated by tribo-electrification and electrostatic induction when different materials are brought in contact with each other. Therefore, it can be applied to various small electronic devices easily, with high efficiency and low costs. ${ }^{\mathbf{1 4 - 1 7}}$ Most materials can be implemented in various ways for generating triboelectricity.

A triboelectric nanogenerator (TENG) consists of two materials, which are oppositely charged, and an electrode that generates external current. TENG has a simple structure and can be developed in various forms using a simple manufacturing process. TENG has been developed in various types to suit different applications or necessities, and can be manufactured as a self-powered device that can be used directly on the human body. ${ }^{18-21}$ Among TENGs, fiber-based triboelectric nanogenerators (FTENG) can convert mechanical energy generated from body motion into electrical energy and can be easily integrated with clothing. ${ }^{22,23}$ In the previous studies, most FTENGs generated electrical output from the contact between two or more strands of thread. However, it was difficult to form contact and separation between threads, and thus, they exhibited slightly low electrical outputs. ${ }^{24}$ Moreover, the previous FTENGs had trouble in bending and it was hard to make stretchable structures for obtaining an effective electrical output, using metal as the electrode material. Additionally, there was limited scope for weaving. ${ }^{25}$

In this paper, we present the development of a new type of fiber-based single-electrode triboelectric nanogenerator (FSTENG) that is composed of stretchable silicone rubber and
IT Fusion Technology Research Center and Department of IT Fusion Technology, Chosun University, 309 Pilmun-daero, Dong-gu, Gwangju 61452, Republic of Korea. E-mail: petruskim@chosun.ac.kr; Tel: +82-62-230-6574

$\dagger$ Electronic supplementary information (ESI) available. See DOI: $10.1039 / \mathrm{c} 7 \mathrm{ra10285b}$ 
conductive thread. We also confirm its electrical efficiency, stability, and durability. FSTENG uses silicone rubber as the negative part and a conductive thread as the electrode. The FSTENG can obtain electrical energy through skin contact. To evaluate the performance of the FSTENG, several strands of FSTENG were fabricated into a woven structure $(45 \mathrm{~mm} \times 45$ $\mathrm{mm}$ ), and were used to charge commercial capacitors and drive LEDs and electronic watches. It was found that, because the FSTENG is stretchable, it is convenient to wear, and it can be fabricated in large sizes. The FSTENG proposed in this letter is expected to be applicable in various products such as wearable and portable devices, owing to its simple manufacturing process and flexibility.

\section{Experimental details}

Fig. 1a shows the schematic and manufacturing process of the FSTENG. An FSTENG is a single-electrode triboelectric generator with a core-shell structure consisting of silicone rubber and conductive thread. The conductive thread, which provides high conductivity and electrical stability, is composed of silvercoated copper and polyester and has an average resistance of $0.049 \Omega \mathrm{cm}^{-1}$ for a diameter of $300 \mu \mathrm{m}$. It exhibits higher mechanical durability, flexibility, and electrical conductivity, compared to other threads coated with metal. ${ }^{26,27}$ In addition, it demonstrates $980 \%$ elongation and has outstanding electrical properties, and hence, is used as a triboelectric material, i.e., the negative part.

In order to fabricate a silicone rubber frame, an elastomer and a curing agent are mixed in a ratio of $1: 1$. The conductive thread is coiled around the silicone rubber frame and it serves as a flexible single electrode. Next, the entire surface is coated with silicone rubber by the dip-coating method, followed by drying at room temperature for approximately three hours, to use it as a material with electric charge. The thickness of the silicone rubber layer on the surface is $100 \mu \mathrm{m}$. In this process, the silicone rubber is cured in vacuum to prevent the electrical resistance from increasing because of the formation of bubbles. Fig. 1b shows a field emission scanning electron microscope (FESEM) photograph of an FSTENG with a diameter of $1.2 \mathrm{~mm}$, and its enlarged side image. This image shows the structure of the silicone rubber frame with the conductive thread coiled around it and a silicone rubber coating on the surface of the fiber. Fig. 1c shows the initial state of the FSTENG and its stretched state under $100 \%$ strain. Even when the length of the FSTENG is increased, the electrical pathway along the conductive thread of the electrode remains constant.

\section{Results and discussion}

A TENG has various modes depending on the contact method of the device and the position of the electrode. ${ }^{15}$ The singleelectrode mode uses electrodes only on one material, out of the two different materials that are in contact. This mode has a structure that changes free-moving motion into electric current, and generates current in the course of maintaining the balance of electric charges, through the movement of electrons from the electrodes according to the contact or separation of the charged substances. This single-electrode mode has the

\section{a}
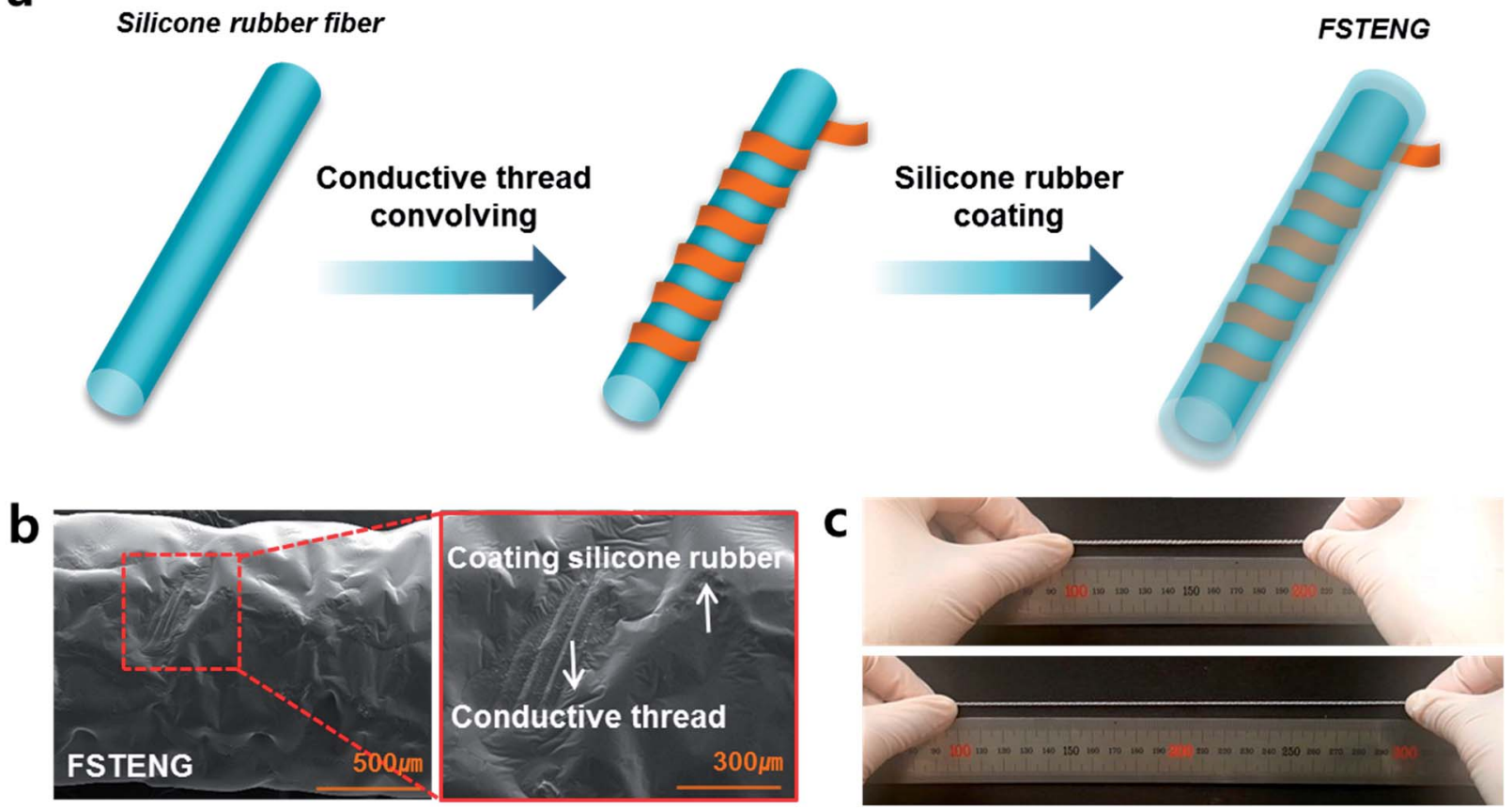

Fig. 1 (a) Schematic of the structure and two step manufacture process for the stretchable FSTENG. The FSTENG is composed of silicone rubber and conductive thread. (b) FESEM image of FSTENG with a diameter and enlarged side image. (c) Photograph of the FSTENG, released state and stretched state with $100 \%$ strain. 


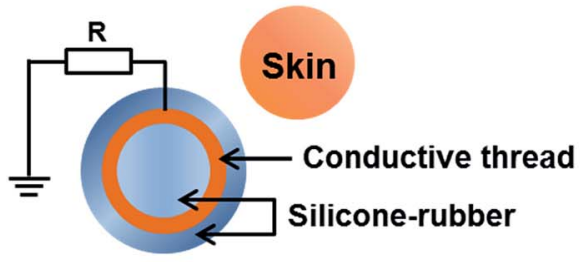

a
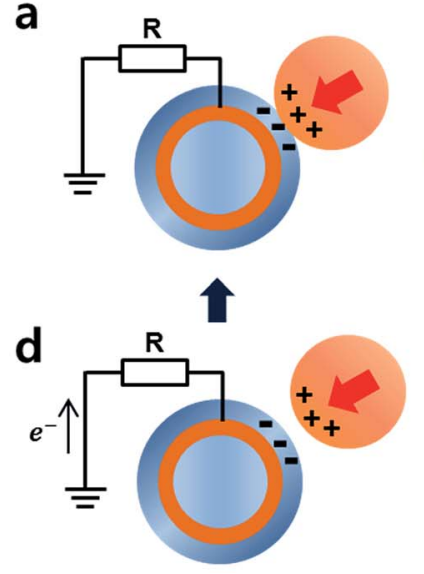

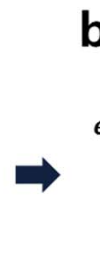

b

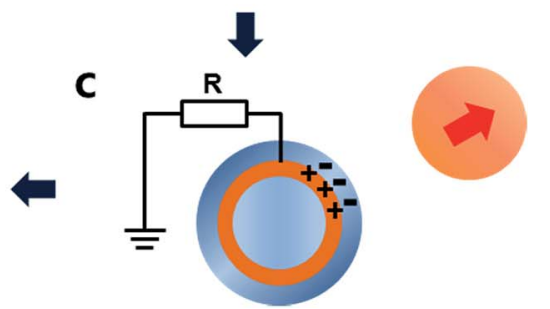

Fig. 2 Schematic the charge distribution of the FSTENG under contact between human skin and FSTENG. (a) Contact between human skin and FSTENG. (b) Human skin and FSTENG are separated by small distance. (c) Increasing distance between human skin and FSTENG. (d) Two separated surfaces are approaching each other.

advantage that the movements of the other materials are not limited.

The power generation process in the FSTENG is illustrated in Fig. 2. The FSTENG generates electrical output using a combination of triboelectric and electrostatic induction in different materials during regular contact and noncontact. As human skin and silicone rubber have different features, they cause the movement of surface charges when they are in contact with each other. According to the triboelectric series, the electrons move
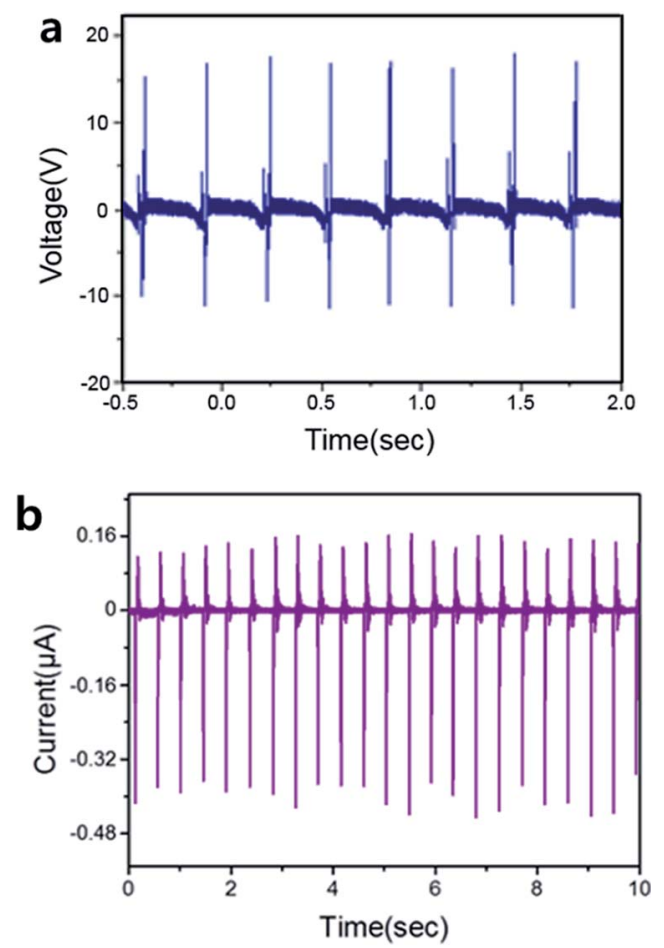

Fig. 3 Electrical characteristics of the FSTENG. (a) Open-circuit voltage and (b) short-circuit current of the FSTENG under a force of 1 kgf. from human skin to silicone rubber during contact because silicone rubber has a higher electron affinity than human skin (Fig. 2a). When the two surfaces are separated, the negative charge on the silicone rubber surface induces a positive charge in the conductive thread, causing current to flow from human skin to the conductive thread (Fig. 2b). This electrostatic induction provides electrical current flow, through the electron mobility. When the negative triboelectric charge on silicone rubber is
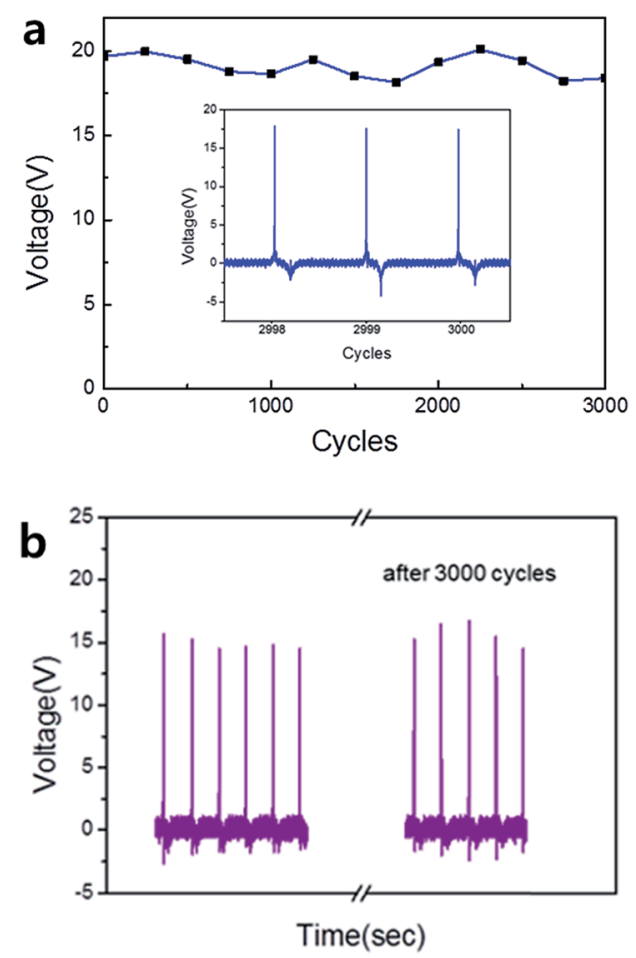

Fig. 4 Electrical stability and durability of the FSTENG. (a) Performance during pushing 3000 cycles using pushing tester under a force of 0.6 kgf. (b) Performance before and after stretching by $50 \% 3000$ cycles using tension tester. 
balanced by the induced charge, no electrical output is observed (Fig. 2c). When human skin and silicone rubber come in contact again, the induced positive electric charge of the conductive thread decreases and a reverse current is generated (Fig. 2d). As a result, an electrical output is generated by the continuous contact and separation of human skin and silicone rubber.

In FSTENG, silicone rubber is a triboelectric layer that generates electrical output through friction with human skin. The electrical characteristics are analyzed by bringing an FSTENG $8.5 \mathrm{~cm}$ in length and $1.2 \mathrm{~mm}$ in diameter in contact with human skin. The output voltage is measured by connecting an electrode to the output of an oscilloscope (MSO9104A), and the short-circuit current is measured using a precision source/ measure device (B2911A). When the skin and FSTENG are in contact with a force of $1 \mathrm{kgf}$, the instantaneous voltage and current signals can be measured. The maximum output voltage and current are $28 \mathrm{~V}$ and $0.56 \mu \mathrm{A}$, as shown in Fig. 3a and b, respectively.

In order to supply energy to wearable devices, it is necessary for the FSTENG to generate energy and be flexible, durable, and stable. As shown in Fig. 4a, the durability is analyzed by the repeated application of pressure using a pushing tester (JIPT-
100). A constant output of $90 \%$ of the electrical output generated under normal operating conditions, when compared to the initial output voltage, is obtained from the FSTENG when a force of $0.6 \mathrm{kgf}$ is applied for 3000 pushing cycles. Fig. 4b, which shows the comparisons of the FSTENG before and after the stretching tests wherein it was stretched by $50 \% 3000$ times using a tensile tester, shows that a stable triboelectric performance is obtained. Thus, it can be concluded that the FSTENG shows outstanding durability without degrading the electrical output.

In wearable energy harvesting, the most easily available energy source is used with clothing. Thus, energy can be harvested most efficiently from the human motion in daily life. Therefore, we fabricated a $45 \mathrm{~mm} \times 45 \mathrm{~mm}$ woven structure by weaving several strands of the FSTENG proposed in our work, and analyzed its electrical characteristics (Fig. 5a). As shown in Fig. $5 \mathrm{~b}$ and $\mathrm{c}$, maximum voltage and current values of $170 \mathrm{~V}$ and $6 \mu \mathrm{A}$ are obtained, and these are six and ten times the voltage and current from one strand of the FSTENG. Thus, the charging performance can be improved by increasing the tribo-surface area and efficiency. It is possible to manufacture various products such as smart clothes using the FSTENG, because FSTENG a

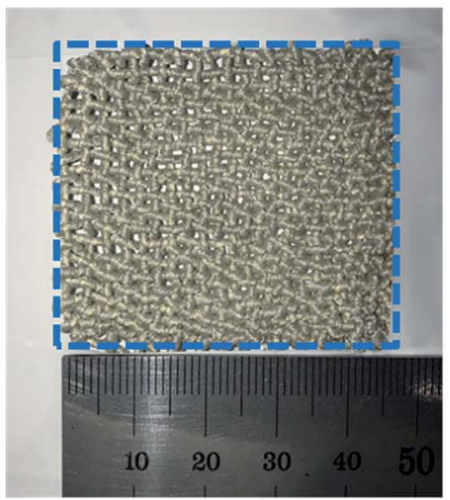

b

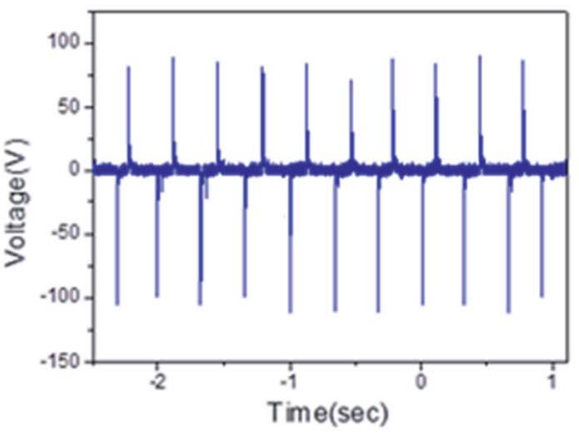

C

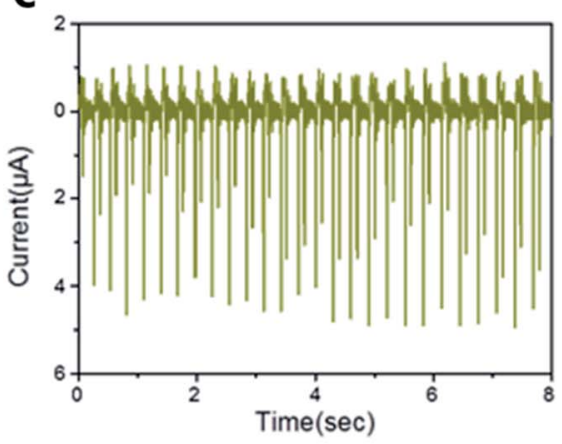

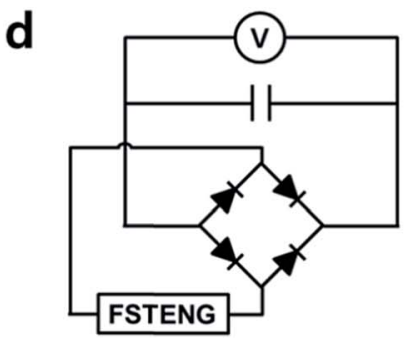

e
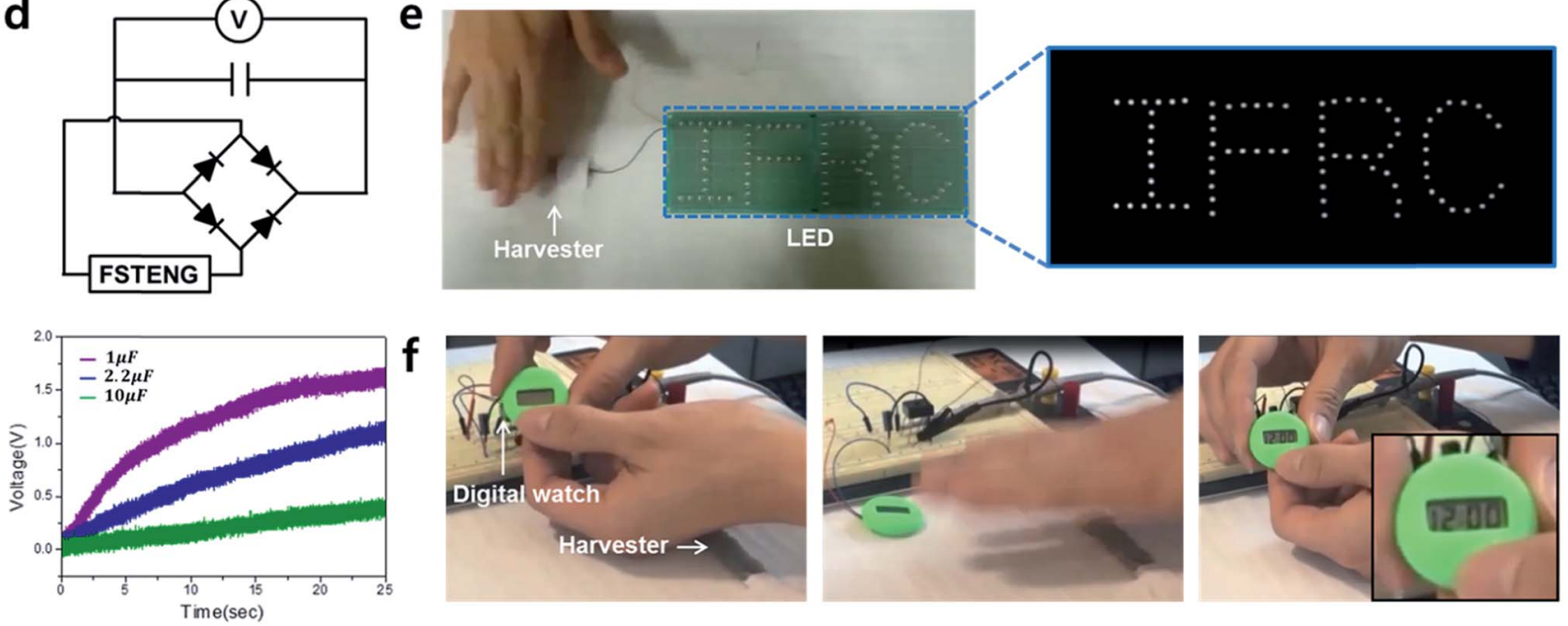

Fig. 5 (a) Photograph of the FSTENG based woven structure $(45 \mathrm{~mm} \times 45 \mathrm{~mm}$ ). (b) Electrical open-circuit voltage and (c) short-circuit current. (d) Circuit diagram of a full-wave bridge rectifier and charging curve of different capacitors. (e) Light of 82 white LEDs and visible in dark environment. (f) The digital watch was lit up by the FSTENG based woven structure. 
can be fabricated easily and it is possible to use it to supply energy easily to wearable devices.

Because the FSTENG based woven structure generates power from alternating current (AC), it is confirmed that a $2.2 \mu \mathrm{F}$ capacitor is charged up to $1.2 \mathrm{~V}$ in $25 \mathrm{~s}$ using a bridge rectifier circuit that converts AC to direct current (DC) (Fig. 5d). The capacitors can accumulate the charges until the positive and negative charges are parallel to the externally applied voltage. According to the formula $Q=C V$, the amount of charge stored in the $2.2 \mu \mathrm{F}$ capacitor over $25 \mathrm{~s}$ is approximately $2.64 \mu \mathrm{C}$. The voltages measured at both ends of capacitors of various capacitances increase with time, and it is confirmed that the device successfully outputs the electric energy. Through this outstanding power generation performance, we can confirm the practical applications. To verify the LED operation, we use a white commercial LED that requires a minimum voltage of $1.8 \mathrm{~V}$ and a current of $100 \mu \mathrm{A}$. Since the FSTENG based woven structure can generate enough electrical output voltage above $100 \mathrm{~V}, 82 \mathrm{LEDs}$ are connected in series and driven. Fig. 5e shows the letters "IFRC" formed by LED lights that are lit by contacting the human skin with an FSTENG based woven structure. In addition, the possibility of running an electronic watch through continuous skin contact is shown in Fig. 5f. The live demonstration of the process of running an electronic watch, in ESI Video $\mathrm{S} 1, \dagger$ confirms the FSTENG's potential for self-powered operation. Therefore, it is shown that it is easy to fabricate the proposed FSTENG as a woven structure and that energy can be generated by the triboelectric effect with human skin. The FSTENGs, as self-powered devices, can be used to supply energy to small electronic devices such as wearable devices, by efficiently converting the mechanical energy generated by human motion into electrical energy.

\section{Conclusions}

A highly stretchable FSTENG was fabricated using silicone rubber and conductive thread. The advantages of the FSTENG proposed in this paper, when compared to the previously studied FTENGs, are: (1) 100\% high stretchability and flexibility; (2) demonstration of durability and stability without deterioration of output, even with repetitive external force; (3) suitability of being fabricated in two-dimensional large areas, which indicates that the power generation performance can be improved by widening the tribo-surface. In the proposed FSTENG, energy was generated from the contact between human skin and silicone rubber. The output voltage and current were $28 \mathrm{~V}$ and $0.56 \mu \mathrm{A}$, respectively. A woven structured $(45 \mathrm{~mm} \times 45 \mathrm{~mm})$ energy harvester was fabricated using several strands of the FSTENG, and voltage and current of $170 \mathrm{~V}$ and 6 $\mu \mathrm{A}$, respectively, were obtained through the contact with human skin. The fabricated FSTENG based woven structure proved that it could be used in self-powered systems, by driving 82 white commercial LEDs and an electronic watch. The proposed FSTENG is expected to provide a new solution for supplying power to wearable devices through human motion based on stretchable textiles of large dimensions.

\section{Conflicts of interest}

There are no conflicts to declare.

\section{Acknowledgements}

This research was supported by the Ministry of Science and ICT (MSIT), Korea, under the Information Technology Research Center (ITRC) support program (IITP-2017-2015-0-00390) supervised by the Institute for Information \& communications Technology Promotion (IITP).

\section{Notes and references}

1 V. S. Mallela, V. Ilankumaran and N. S. Rao, Indian Pacing Electrophysiol. J., 2004, 4, 201.

2 R. Riemer and A. Shapiro, J. NeuroEng. Rehabil., 2011, 8, 22. 3 F. R. Fan, W. Tang and Z. L. Wang, Adv. Mater., 2016, 28, 4283. 4 S. P. Beeby, M. J. Tudor and N. M. White, Meas. Sci. Technol., 2006, 17, 175.

5 C. Dagdeviren, B. D. Yang, Y. Su, P. L. Tran, P. Joe, E. Anderson, J. Xia, V. Doraiswamy, B. Dehdashti, X. Feng, B. Lu, R. Poston, Z. Khalpey, R. Ghaffari, Y. Huang, M. J. Slepian and J. A. Rogers, Proc. Natl. Acad. Sci. U. S. A., 2014, 111, 1927.

6 C. Dagdeviren, S.-W. Hwang, Y. Su, S. Kim, H. Cheng, O. Gur, R. Haney, F. G. Omenetto, Y. Huang and J. A. Rogers, Small, 2013, 9, 3398.

7 Z. L. Wang, ACS Nano, 2013, 7, 9533.

8 Z. L. Wang, J. Chen and L. Lin, Energy Environ. Sci., 2015, 8, 2250.

9 C. J. Lee, A. Y. Choi, J. Park, C. Choi, H. J. Sim, S. J. Kim and Y. T. Kim, RSC Adv., 2016, 6, 10094.

10 V. Leonov and R. J. M. Vullers, J. Electron. Mater., 2009, 38, 1491.

11 Y. K. Ramadass and A. P. Chandrakasan, IEEE J. Solid-State Circuits, 2011, 46, 333.

12 S. P. Beeby, R. N. Torah, M. J. Tudor, P. Glynne-Jones, T. O'Donnell, C. R. Saha and S. Roy, Journal of Micromechanics and Microengineering, 2007, 17, 1257.

13 P. Li, S. Gao and H. Cai, Microsyst. Technol., 2015, 21, 401.

14 G. Zhu, C. Pan, W. Guo, C. Y. Chen, Y. Zhou, R. Yu and Z. L. Wang, Nano Lett., 2012, 12, 4960.

15 Z. L. Wang, RSC Adv., 2014, 176, 447.

16 S. Lee, W. Ko, Y. Oh, J. Lee, G. Baek, Y. Lee, J. Sohn, S. Cha, J. Kim, J. Park and J. Hong, Nano Energy, 2015, 12, 410.

17 A. Y. Choi, C. J. Lee, J. Park, D. Kim and Y. T. Kim, Sci. Rep., 2017, 7, 45583.

18 X. Pu, L. Li, H. Song, C. Du, Z. Zhao, C. Jiang, G. Cao, W. Hu and Z. L. Wang, Adv. Mater., 2015, 27, 2472.

19 W. Yang, J. Chen, G. Zhu, J. Yang, P. Bai, Y. Su, Q. Jing, X. Cao and Z. L. Wang, ACS Nano, 2013, 7, 11317.

20 F. Yi, X. Wang, S. Niu, S. Li, Y. Yin, K. Dai, G. Zhang, L. Lin, Z. Wen, H. Guo, J. Wang, M.-H. Yeh, Y. Zi, Q. Liao, Z. You, Y. Zhang and Z. L. Wang, Sci. Adv., 2016, 2, e1501624.

21 Y. Zi, S. Niu, J. Wang, Z. Wen, W. Tang and Z. L. Wang, Nat. Commun., 2015, 6, 8376. 
22 P. Xu, T. Gu, Z. Cao, B. Wei, J. Yu, F. Li, J. H. Byun, W. Lu, Q. Li and T. W. Chou, Adv. Energy Mater., 2014, 4, 1300759.

23 X. He, Y. Zi, H. Guo, H. Zheng, Y. Xi, C. Wu, J. Wang, W. Zhang, C. Lu and Z. L. Wang, Adv. Funct. Mater., 2017, 27, 1604378.

24 J. Zhong, Y. Zhang, Q. Zhong, Q. Hu, B. Hu, Z. L. Wang and J. Zhou, ACS Nano, 2014, 8, 6273.
25 X. Li, Z.-H. Lin, G. Cheng, Z. Wen, Y. Liu, S. Niu and Z. L. Wang, ACS Nano, 2014, 8, 10674.

26 Y.-C. Lai, J. Deng, S. L. Zhang, S. Niu, H. Guo and Z. L. Wang, Adv. Funct. Mater., 2017, 27, 1604462.

27 J. Ge, L. Sun, F.-R. Zhang, Y. Zhang, L.-A. Shi, H.-Y. Zhao, H.-W. Zhu, H.-L. Jiang and S.-H. Yu, Adv. Mater., 2016, 28, 722. 